\title{
Static and Dynamics of Topological Defects in Magnetic Dots*
}

\author{
J.-C.S. LÉVY \\ Laboratoire Matériaux et Phénomènes Quantiques, CNRS UMR 7162 \\ Université Paris 7-Denis Diderot, 10 rue Alice Domont et Léonie Duquet, 75013 Paris, France
}

(Received May 24, 2008)

\begin{abstract}
A Taylor expansion of dipole-dipole interaction in 2D systems defines a Landau-like local dipolar interaction in spin derivative field. The lowest order of this interaction gives the dipolar anisotropy. The next non-zero order is responsible for the appearance of magnetic vortices and hyperbolic defects. The following non-zero orders indicate the occurrence of higher topological defects such as double circle and of modulations. The arrangement of self-screened topological defects is discussed in agreement with the Monte Carlo simulations and experimental observations. Excited localized modes associated with these defects are classified.
\end{abstract}

PACS numbers: 75.60.Ch, 75.75.+a

\section{Introduction}

Recently, the improvement of resolution of $2 \mathrm{D}$ magnetic structures enabled several authors to observe vortices and other topological defects in magnetic dots with weak anisotropy $[1-5]$. This is in agreement with the numerical observation of vortex stability in 2D samples with dipole-dipole interaction by the Monte Carlo techniques [6], as well as by micromagnetic computations [7-9]. It must be added that the geometrical arrangement of vortices on $2 \mathrm{D}$ lattices naturally requires the occurrence of other competing topological defects. Quite similarly improved resolution in resonance techniques enabled people to observe magnetic vortex resonance [10]. Therefore, the purpose of a natural classification of topological defects in 2D quantum dots and of their excitation modes from the very nature of dipole-dipole interaction, which is the goal of this paper, just arises. The idea behind such a tentative classification is just a TaylorMaclaurin series expansion of dipolar interactions which enables us to define a Landau-like local version of the Hamiltonian as a function of the spin field and its derivatives. So, the ground state determination is achieved by optimizing these different contributions. This defines classified structures which are topological defects and further this Hamiltonian enables us to classify their excitations. Moreover, in presence of strong perpendicular anisotropy, this calculation explains the occurrence of observed labyrinthine domains in 2D dots [11] and gives other evidence for the trace of topological defects. Quite similarly this calculation suggests a normal classification of excitations in labyrinthine structures.

\footnotetext{
* Proceedings of the European Conference "Physics of Magnetism", Poznań 2008.
}

\section{Local picture of dipolar interaction}

The usual dipolar Hamiltonian reads [12]:

$$
H_{\mathrm{d}}=\sum_{i, j \neq i} \frac{\boldsymbol{S}_{i} \cdot \boldsymbol{S}_{i}}{r_{i j}^{3}}-3 \sum_{i, j \neq i} \frac{\left(\boldsymbol{S}_{i} \cdot \boldsymbol{r}_{i j}\right)\left(\boldsymbol{S}_{i} \cdot \boldsymbol{r}_{i j}\right)}{r_{i j}^{5}} .
$$

Here a part of a discrete triangular or square 2D lattice of spins $\boldsymbol{S}_{i}$ located at sites $i$ of coordinates $\left(x_{i}, y_{i}\right)$ fills the dot with the running vector $\boldsymbol{r}_{i j}$ linking sites $i$ and $j$. The Taylor expansion of the lattice spin field at site $j$ reads as a function of the spin field at site $i$ and of its partial derivatives

$$
\boldsymbol{S}_{j}=\sum_{p, q=0}^{\infty, \infty} \frac{x_{i j}^{p} y_{i j}^{q}}{p ! q !}\left(\frac{\partial^{p+q} \boldsymbol{S}_{i}}{\partial x^{p} \partial y^{q}}\right) .
$$

Using this expansion, two kinds of lattice sums appear in Eq. (1), namely the isotropic sum $I_{p, q}$ and the anisotropic sum $J_{p, q, \alpha, \beta}$ with

$$
I_{p, q}=\sum_{j}^{\prime} \frac{x_{i j}^{p} y_{i j}^{q}}{p ! q !\left(x_{i j}^{2}+y_{i j}^{2}\right)^{3 / 2}}
$$

and

$$
J_{p, q, \alpha, \beta}=\sum_{j}{ }^{\prime} \frac{\left(\boldsymbol{r}_{i j}\right)_{\alpha}\left(\boldsymbol{r}_{i j}\right)_{\beta} x_{i j}^{p} y_{i j}^{q}}{p ! q !\left(x_{i j}^{2}+y_{i j}^{2}\right)^{5 / 2}} .
$$

These lattice sums depend up to some extent on lattice symmetry [13], and some of them do not converge for an infinite lattice without damping of the dipolar interaction. Therefore, the local dipolar Hamiltonian reads as a sum of local Hamiltonians up to a corrective term

$$
H_{\mathrm{D}}=\sum_{i} H_{\mathrm{d}, i},
$$

where 


$$
\begin{aligned}
H_{\mathrm{d}, i} & =\sum_{p, q=0}^{\infty, \infty} \boldsymbol{S}_{i} \cdot\left(\frac{\partial^{p+q} \boldsymbol{S}_{i}}{\partial x^{p} \partial y^{q}}\right) I_{p, q} \\
-3 & \sum_{p, q=0}^{\infty, \infty}\left(\boldsymbol{S}_{i}\right)_{\alpha}\left(\frac{\partial^{p+q}\left(\boldsymbol{S}_{i}\right)_{\beta}}{\partial x^{p} \partial y^{q}}\right) J_{p, q, \alpha, \beta} .
\end{aligned}
$$

\section{Ground state determination}

The lowest order term of Eq. (5) is responsible for lattice induced anisotropy [13]. The next non-zero terms are even terms because of symmetry. The first of these terms reads

$$
\begin{gathered}
\frac{H_{2}}{C}=-5 S_{x} \frac{\partial^{2} S_{x}}{\partial x^{2}}+3 S_{y} \frac{\partial^{2} S_{y}}{\partial x^{2}}+3 S_{x} \frac{\partial^{2} S_{x}}{\partial y^{2}} \\
-5 S_{y} \frac{\partial^{2} S_{y}}{\partial y^{2}}-6 S_{x} \frac{\partial^{2} S_{y}}{\partial x \partial y}-6 S_{y} \frac{\partial^{2} S_{x}}{\partial x \partial y} .
\end{gathered}
$$

This is already a non-linear term. The next non-zero term is also non-linear and reads

$$
\begin{gathered}
\frac{H_{4}}{C^{\prime}}=-9 S_{x} \frac{\partial^{4} S_{x}}{\partial x^{4}}+3 S_{y} \frac{\partial^{4} S_{y}}{\partial x^{4}}-6 S_{x} \frac{\partial^{4} S_{x}}{\partial x^{2} \partial y^{2}} \\
-6 S_{y} \frac{\partial^{4} S_{y}}{\partial x^{2} \partial y^{2}}+3 S_{x} \frac{\partial^{4} S_{x}}{\partial y^{4}}-9 S_{y} \frac{\partial^{4} S_{y}}{\partial y^{4}} \\
-12 S_{x}\left(\frac{\partial^{4} S_{y}}{\partial x^{3} \partial y}+\frac{\partial^{4} S_{y}}{\partial y^{3} \partial x}\right) \\
-12 S_{x}\left(\frac{\partial^{4} S_{x}}{\partial x^{3} \partial y}+\frac{\partial^{4} S_{x}}{\partial y^{3} \partial x}\right) .
\end{gathered}
$$

Here $C$ and $C^{\prime}$ are positive constants. For a basically in-plane spin $\boldsymbol{S}=(\cos \theta, \sin \theta, 0)$ as observed in experiments $[1-5]$ and in numerical simulations [6-9], these contributions become respectively

$$
\begin{aligned}
& H_{2} \approx 4 \sin 2 \theta\left(\theta_{x^{2}}-\theta_{y^{2}}\right)-6 \cos 2 \theta \theta_{x y}+\theta_{x}^{2}+\theta_{x}^{2} \\
& \quad+6 \sin 2 \theta \theta_{x} \theta_{y}+4 \cos 2 \theta\left(\theta_{x}^{2}-\theta_{x}^{2}\right)
\end{aligned}
$$

and

$$
\begin{aligned}
& H_{4} \approx 2 \sin 2 \theta\left[\theta_{x^{4}}-\theta_{y^{4}}+2\left(\theta_{x^{3}} \theta_{y}+\theta_{y^{4}} \theta_{x}\right)\right. \\
& +4 \theta_{x y}\left(3 \theta_{x^{2}}+3 \theta_{y^{2}}-2 \theta_{x} \theta_{y}\right)+6\left(\theta_{x^{2} y}+\theta_{y^{2} x}\right) \\
& +6\left(\theta_{x^{2}} \theta_{x}^{2}-\theta_{y^{2}} \theta_{y}^{2}\right)-2 \theta_{x} \theta_{y}\left(\theta_{x}^{2}+\theta_{y}^{2}\right) \\
& \quad-4 \cos 2 \theta\left(\theta_{x^{3} y}+\theta_{x y^{3}}\right) \\
& +\left(4 \cos ^{2} \theta-1\right)\left(4 \theta_{x^{3}} \theta_{x}+3 \theta_{x^{2}}^{2}-\theta_{x}^{4}\right) \\
& +\left(4 \sin ^{2} \theta-1\right)\left(4 \theta_{y^{3}} \theta_{y}+3 \theta_{y^{2}}^{2}-\theta_{y}^{4}\right) \\
& +4\left(\theta_{x^{2} y} \theta_{y}+\theta_{x y^{2}} \theta_{x}\right)+2 \theta_{x^{2}} \theta_{y^{2}}+4 \theta_{x y}^{2} \\
& -12 \theta_{x y}\left(\theta_{x^{2}}+\theta_{y^{2}}\right)-12 \theta_{x} \theta_{y}\left(\theta_{x^{2}}+\theta_{y^{2}}\right)-2 \theta_{x}^{2} \theta_{y}^{2} .
\end{aligned}
$$

These equations are local, but strongly non-linear.
Then it is convenient to search for optimal structures in polar coordinates $\theta(r, \varphi)$ [14], and more specifically to look at vortex-like solutions which do not depend on the radial distance. Therefore, the previous contributions read now

$$
\frac{H_{2}}{A_{2,0}} \approx \frac{\theta_{\varphi}^{2}}{2 r^{2}}[2-\cos (2(\varphi+\theta))-7 \cos (2(\varphi-\theta))]
$$

and

$$
\begin{aligned}
& \frac{H_{4}}{A_{4,0}} r^{4} \approx \theta_{\varphi}[-6 \cos (2(\theta-\varphi))-2 \cos (2(\theta+\varphi))] \\
& \quad+\theta_{\varphi}^{2}[13+46 \cos (2(\theta-\varphi))+26 \cos (2(\theta+\varphi)) \\
& \quad+11 \cos (4 \varphi)]+\theta_{\varphi}^{3}[16 \cos (2(\theta-\varphi))-6 \cos (2(\theta+\varphi)) \\
& \quad-\cos (4 \varphi-2 \theta)+\cos (4 \varphi+2 \theta)+12 \cos (2 \varphi)] \\
& \quad+\theta_{\varphi}^{4}\left[1+\cos (2(\theta-\varphi))-\cos (2(\theta+\varphi))-1.5 \sin ^{2}(2 \varphi)\right. \\
& \left.\quad-\left(\sin ^{4} \varphi \cos ^{2} \theta+\cos ^{4} \varphi \sin ^{2} \theta\right)\right] .
\end{aligned}
$$

Tentative ground state solutions must optimize these contributions and their energy is compared with the ones for uniform ferromagnetic structure $\theta=$ const.

\section{Topological defects as optimal structures}

From Eq. (8), vortices $\theta=\varphi \pm \frac{\pi}{2}(2 \pi): \mathrm{V}_{1}, \mathrm{~V}_{2}$ and hyperbolic defects $\theta=-\varphi \pm \frac{\pi}{2}(2 \pi): \mathrm{H}_{1}, \mathrm{H}_{2}$ as shown from the field lines $\frac{\mathrm{d} r}{r \mathrm{~d} \varphi}=\cot (\theta-\varphi)$ schematically reproduced in Fig. 1, are metastable states at level two. As a matter of fact, the dipolar interaction is damped by the occurrence of defects. This damping stabilizes these topological defects which define so the ground state.
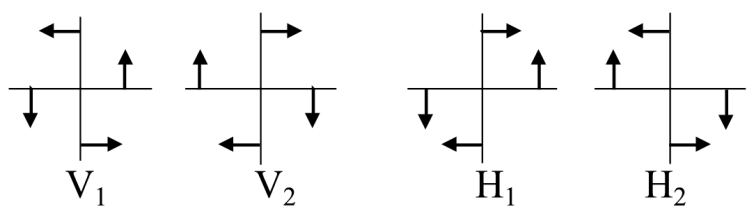

Fig. 1. Schematic representation of the four basic topological defects: vortex $1\left(\mathrm{~V}_{1}\right)$, vortex $2\left(\mathrm{~V}_{2}\right)$, hyperbolic defect $1\left(\mathrm{H}_{1}\right)$ and hyperbolic defect $2\left(\mathrm{H}_{2}\right)$.

These defects were already observed experimentally [5] and can be seen in simulations [6]. Some other names are also used as magnetic flowers for hyperbolic defect [9]. In the case of strong perpendicular anisotropy, this explains the stability of maze structures.

At level four as given by Eq. (9), these vortices and hyperbolic defects also appear as metastable as well as other topological defects characterized by the equations which initially appeared about liquid crystals for Frank's disclinations [15]: 


$$
\begin{aligned}
\theta & =2 \varphi(\pi), \\
\theta & =2 \varphi+\frac{\pi}{2}(\pi), \\
\theta & =-2 \varphi(\pi), \\
\theta & =-2 \varphi+\frac{\pi}{2}(\pi) .
\end{aligned}
$$

The corresponding field lines define a double circle DC and a sextic curve $r=r_{0}\|\cos 3 \varphi\|^{1 / 3} \mathrm{HH}$ as shown schematically in Fig. 2. These defects can be distinguished in simulated Monte Carlo systems [6]. In the case of strong perpendicular anisotropy, this explains the arrangement of adjacent maze structures. The extrapo-

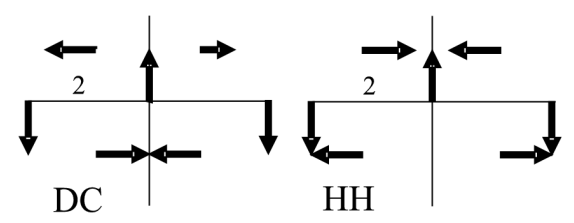

Fig. 2. Schematic view of the two basic fourth order topological defects: $\mathrm{DC}$ - the double circle and $\mathrm{HH}-$ the sextic curve.

lation to higher orders gives the following disclinations:

$$
\theta= \pm n \varphi(2 \pi) .
$$

In the case of strong perpendicular anisotropy, this explains the arrangement of several maze structures together.

\section{Defect dynamics and conclusions}

A first excitation mode for a defect consists in the whole motion of this defect which can be a vortex, a hyperbolic defect or other associations of vortices as DC and HH. A second defect mode consists in a "breathing" mode as usual for localized defects. This mode with successive increase and shrinking of its area is obviously coupled with the motions of other close defects. Then the quartic modulation of Eq. (9) is also responsible for quartic deformations of these defects. The extrapolation to higher order means also a sextic deformation and so on. This defines more complex vibrational modes of the defects. Finally, creation and annihilation of defects must also occur. Therefore, quite numerous excitation modes are expected to occur and their inertial contributions are also different. Quite obviously, a similar classification of maze excitations occurs in the case of strong perpendicular anisotropy.

As a conclusion, there is a link between topological defects in magnetic dots and topological defects in liquid crystals. There is also an efficient screening of dipolar interaction by topological defects and this is that screening which is responsible for the observation of networks of topological defects as they are in magnetic dots [1-5]. Quite numerous modes of various symmetry are expected to occur.

\section{References}

[1] A. Vaterlaus, C. Stamm, U. Maier, M.G. Pini, P. Politi, D. Pescia, Phys. Rev. Lett. 84, 2247 (2000).

[2] S.P. Li, D. Peyrade, M. Natali, A. Lebib, Y. Chen, U. Ebels, L.D. Buda, K. Ounadjela, Phys. Rev. Lett. 86, 1102 (2001); S.P. Li, M. Natali, A. Lebib, A. Pepin, Y. Chen, Y.B. Xu, J. Magn. Magn. Mater. 241, 447 (2002).

[3] T. Shinjo, T. Okuno, R. Hassdorf, K. Shigeto, T. Ono, Science 289, 930 (2000).

[4] T. Duden, E. Bauer, Phys. Rev. Lett. 77, 2308 (1996); M. Hehn, R. Ferre, K. Ounadjela, J.-P. Bucher, F. Rousseaux, J. Magn. Magn. Mater. 165, 5 (1997).

[5] J. Li, C. Rau, Phys. Rev. Lett. 97, 107201 (2006); J.E. Villegas, C.-P. Li, I.K. Schuller, Phys. Rev. Lett. 99, 227001 (2007).

[6] E.Y. Vedmedenko, A. Ghazali, J.-C.S. Levy, Surf. Sci. 402-404, 391 (1998); Phys. Rev. B 59, 3329 (1999); E.Y. Vedmedenko, H.P. Oepen, A. Ghazali, J.-C.S. Levy, J. Kirschner, Phys. Rev. Lett. 84, 5884 (2000); J. Sasaki, F. Matsubara, J. Phys. Soc. Japan 66, 2138 (1997).

[7] J.-G. Zhu, H.N. Bertram, J. Appl. Phys. 63, 3248 (1988); I.R. McFadyen, I.A. Beardsley, J. Appl. Phys. 67, 5540 (1990).

[8] D.R. Fredkin, T.R. Koehler, J. Appl. Phys. 67, 5544 (1990); D.R. Fredkin, T.R. Koehler, J.F. Smyth, S. Schultz, J. Appl. Phys. 69, 5276 (1991).

[9] R.P. Cowburn, M.E. Welland, Phys. Rev. B 58, 9217 (1998).

[10] R.L. Compton, P.A. Crowell, Phys. Rev. Lett. 97, 137202 (2006).

[11] P. Molho, J. Gouzerh, J.-L. Porteseil, J.-C.S. Levy, J. Magn. Magn. Mater. 54-57, 857 (1986); P. Molho, J.-L. Porteseil, Y. Souche, J. Gouzerh, J.-C.S. Levy, J. Appl. Phys. 61, 4188 (1987); M. Seul, D. Andelman, Science 267, 476 (1995).

[12] L.D. Landau, E.M. Lifshitz, The Classical Theory of Fields, 2nd ed., Pergamon Press, Oxford 1962, p. 119.

[13] J.-C.S. Levy, Phys. Rev. B 63, 104409 (2001).

[14] J.-C.S. Levy, to be published.

[15] F.C. Frank, Disc. Faraday Soc. 25, 19 (1958). 